\title{
Longitudinal disease-associated gut microbiome differences in infants with early food allergic manifestations
}

\section{Victoria Martin}

Harvard Medical School, Massachusetts General Hospital https://orcid.org/0000-0002-4322-9564

\section{Yamini Virkud}

Harvard Medical School, Massachusetts General Hospital

Ehud Dahan

The Hebrew University of Jerusalem https://orcid.org/0000-0002-1204-9413

\section{Hannah Seay}

Massachusetts General Hospital

\section{Hera Vlamakis}

Broad Institute https://orcid.org/0000-0003-1086-9191

\section{Ramnik Xavier}

Massachusetts General Hospital https://orcid.org/0000-0002-5630-5167

\section{Wayne Shreffler}

Harvard Medical School, Massachusetts General Hospital

\section{Qian Yuan}

Massachusetts General Hospital

Moran Yassour ( $\square$ Moran.Yassour@mail.huji.ac.il)

Hebrew University of Jerusalem https://orcid.org/0000-0002-7344-5188

\section{Article}

Keywords: gut microbiome, food allergy, paedatrics, gastrointestinal disease

Posted Date: July 12th, 2021

DOl: https://doi.org/10.21203/rs.3.rs-654476/v1

License: (c) (1) This work is licensed under a Creative Commons Attribution 4.0 International License. Read Full License 
1 Title:

2 Longitudinal disease-associated gut microbiome differences in infants with early 3 food allergic manifestations

\section{Authors:}

5 Victoria M. Martin, MD, MPH; Yamini V. Virkud, MD, MA, MPH, Ehud Dahan, Hannah L. Seay,

6 Hera Vlamakis, PhD, Ramnik Xavier, MD, PhD, Wayne G. Shreffler, MD, PhD, Qian Yuan, MD,

$7 \mathrm{PhD}^{*}$, Moran Yassour, PhD*

8 Abstract ( 150 words):

9 Complex interactions between the gut microbiome and immune cells in infancy are thought to

10 be part of the pathogenesis for the marked rise in pediatric allergic diseases, particularly food

11 allergies $^{1-5}$. Food protein-induced allergic proctocolitis (FPIAP) is commonly the earliest

12 recognized non-lgE-mediated food allergy in infancy and is associated with atopic dermatitis

13 and subsequent IgE-mediated food allergy later in childhood ${ }^{6,7}$. Yet, a large prospective

14 longitudinal study of the microbiome of infants with FPIAP (including samples prior to symptom

15 onset) has not been done. Here we analyzed 954 longitudinal samples from 160 infants in a

16 nested case-control study (81 who developed FPIAP, and 79 matched controls) from 1 week to

171 year of age by $16 \mathrm{~S}$ rRNA ribosomal gene sequencing as part of the Gastrointestinal

18 Microbiome and Allergic Proctocolitis (GMAP) Study. We confirmed that vaginally delivered

19 infants had a greater abundance of Bacteroides, infants who received any breast milk had a

20 greater abundance of Bifidobacterium, and that overall bacterial richness rose over the first

21 year. We found key differences in the microbiome of infants with FPIAP, most strongly a higher

22 abundance of a genus of Enterobacteriaceae and a lower abundance of a family of Clostridiales

23 during the symptomatic period, as well as other key taxonomic differences across symptom

24 states including prior to symptom onset. This study contributes to the larger body of literature

25 examining structural development of the early life gut microbiome and provides a foundation for

26 more mechanistic investigation into the pathogenesis and microbial effects on FPIAP and

27 subsequent food allergic diseases in childhood. 


\section{Introduction:}

Food protein-induced allergic proctocolitis (FPIAP) is a commonly recognized and burdensome form of non-IgE-mediated food allergy of early infancy with rates as high as $17 \%$ recently reported in the US from the GMAP study ${ }^{6}$. FPIAP is typically diagnosed clinically by the presence of bloody and mucoid stools during the first few months of life in a generally healthy infant that resolves with dietary restriction (most commonly of milk protein, and sometimes soy $)^{8}$. FPIAP can be seen in both children who are breastfed and formula fed, but exclusively formula fed infants are at increased risk ${ }^{6}$. The pathophysiology of FPIAP is not well studied or understood. It resolves for most affected patients within the first 12 months of life; however it has been associated with an increased risk of developing both IgE-mediated food allergy $(\operatorname{lgE}-\mathrm{FA})^{7}$ and Eosinophilic Esophagitis ${ }^{9}$. The rate of rise of food allergies strongly implicates environmental factors (antibiotics, diet, and other exposures) resulting in dysbiosis (microbial imbalance) which has been already associated with other forms of pediatric allergy ${ }^{1,2}$. Complex cross-talk between the intestinal microbiome, food antigens, intestinal inflammation, and the innate immune system early in life likely contributes to the mechanisms responsible for either healthy tolerance acquisition or food allergy development ${ }^{3}$. Given the early onset of FPIAP, association with feeding practices and symptoms of the lower (and often also upper) GI tract, we hypothesized that the developing infant microbiome plays a role in FPIAP development and subsequent loss of oral tolerance.

There is growing evidence that the gut microbiome plays a key role in the development of $\lg E$ and non-IgE mediated food allergy ${ }^{10-15}$. Infants with IgE-mediated cow's milk allergy (IgE-CMA) have key taxonomic differences when compared to healthy infant counterparts ${ }^{11,14,15}$.

They have additionally been shown to have taxonomic differences once started on extensively hydrolyzed formula and after achieving tolerance to milk protein, suggesting a role for the microbiome in disease resolution ${ }^{14-16}$. Literature on non-IgE-mediated cow's milk protein allergy has similarly demonstrated taxonomic differences between healthy and allergic infants, infants on an extensively hydrolyzed formula diet, and infants tolerant to milk protein ${ }^{10-13,17,18}$. This literature, however, has been limited by cross sectional design ${ }^{10,11}$, lack of strict diagnostic criteria for non-lgE-CMA ${ }^{12,13}$, detection methods by culture ${ }^{12,13}$, and pooling of non-lgE with 
$57 \operatorname{lgE}-\mathrm{CMA}^{11,15}$. To our knowledge, there is no study that has analyzed samples longitudinally 58 including those collected prior to symptom or disease onset.

59 The Gastrointestinal Microbiome and Allergic Proctocolitis (GMAP) Study is the first 60 prospective observational healthy infant cohort study specifically designed to investigate the 61 epidemiology, clinical presentation, and potential role of the longitudinal microbiome in FPIAP

62 and subsequent IgE-FA ${ }^{7}$. Over 1,000 healthy infants were enrolled at their first pediatrics visit in 63 the first week of life, stool samples were collected very frequently across the first year, and they 64 are being followed clinically with ongoing sample collection through age $18.17 \%$ of these 65 healthy children went on to develop FPIAP $^{6}$ and $6 \%$ went on to develop IgE-FA ${ }^{7}$. This large 66 cohort addresses the many limitations in the literature, as we enrolled infants in an unbiased

67 fashion, sampled infants frequently very early in life, and thus have sampling before, during, and 68 after disease onset. This provided us with a well-matched control population, dense early 69 sampling, and the ability to examine factors which may precede disease onset, herald symptom 70 progression, or be associated with disease resolution.

\section{Results:}

\section{Participant profiles}

73 From the GMAP cohort $^{6}$, we selected 81 infants prospectively diagnosed with food 74 protein-induced allergic proctocolitis (FPIAP) and 79 matched controls with robust fecal 75 microbiome sampling across their first year of life (Figure 1). For infants who developed FPIAP, 76 samples were selected from before symptom onset, during their symptomatic period, and after 77 their symptoms resolved (Figure 1). The median number of samples in the first year per child 78 was 5 [3, 19]. A total of 954 samples met quality control criteria (Methods) when sequenced 79 using 16S ribosomal RNA gene sequencing. Data were analyzed using QIIME2 (see Methods), 80 generating a genus-level composition map for each sample. 45\% were female, 65\% were 81 delivered vaginally, $63 \%$ were initially exclusively breastfed, and $56 \%$ were perinatally exposed

82 to antibiotics (maternal $46 \%$, perinatal infant $3 \%$, or both $7 \%$ ) (Figure 1). No significant 83 differences among any of these factors were noted between infants with FPIAP and controls, 
84 with the exception of the initial infant diet which was more commonly formula in the children who

85 developed FPIAP as previously reported in the cohort at large ${ }^{6}$.

\section{Overall microbiome composition}

87 We first examined the microbiome collectively across all infants, looking for characteristic 88 features of the developing infant gut microbiome in the first year of life. We found a 89 predominance of Bifidobacterium, Bacteroides, Enterbacteriales, and Clostridium at the earliest 90 time points as has been previously shown ${ }^{19-22}$ (Figure 2A). We also saw the characteristic rise

91 in overall microbial richness from birth to age $1^{19,22,23}$ (Figure 2B). Consistent with existing

92 literature ${ }^{24,25}$, we found that infants who were vaginally delivered had a greater abundance of

93 Bacteroides $\left(p=5.25 \times 10^{-7}, \boldsymbol{q}\right.$-value $=\mathbf{5 . 0 6 \times 1 0 ^ { - 6 }}$, coefficient $\left.\mathbf{= 0 . 2 3}\right)$ compared to those delivered

94 by C-section, and infants who were exclusively breastfed had a greater abundance of 95 Bifidobacterium $\left(p=3.82 \times 10^{-4}, q=2.25 \times 10^{-3}, \quad \mathbf{c}=\mathbf{0 . 1 3}\right)$ (Figure 2C). Additionally, infants who 96 received probiotics in the first year (predominantly lactobacillus rhamnosus GG in this cohort) 97 had a greater abundance of Lactobacillus (Figure 2C). Therefore, as we turned our attention to 98 exploring taxonomic features that differentiated infants with FPIAP compared to healthy 99 controls, we included age, mode of delivery, diet, and probiotic exposure in all of our multivariate 100 linear models (Methods) reported here. Only results that met an absolute coefficient threshold 101 of $>0.05$ and q-value $<0.25$ are reported. A complete table of all significant findings are available 102 in Supplemental Table 1.

103 Taxonomic differences between FPIAP cases and controls

104 In examining potential differences in the infant microbiome in children who developed FPIAP 105 compared to those who did not, we first looked for overall differences in community structure 106 across the first year. While the overall first year composition was similar between cases and 107 controls (Supplemental Figure 1 (Composition plots by case status), we did find a greater 108 abundance of a single taxa in symptomatic FPIAP cases. We denote this taxa as 109 Enterobacteriaceae_set1, which is an unclassified genus of Enterobacteriaceae (most likely, 
110 represented by Escherichia; Methods) and is found in greater abundance in symptomatic 111 FPIAP cases $\left(p=2.7 \times 10^{-2}, q=1.13 \times 10^{-1}, c=0.09\right)$ when analyzing all samples (Supplemental

112 Table 1). We did not find any statistically significant differences between the overall microbial

113 richness or stability across the first year when comparing FPIAP cases and controls, using 114 shannon, chao1, and bray-curtis indices, though some pairwise differences were noted at 4 and 1156 months (Supplemental Figure 2) between symptomatic cases and resolved cases and 116 controls respectively.

117 Given that FPIAP presents so early in infancy (median age of diagnosis $=1$ month, $>75 \%$

118 diagnosed by 2.5 months), we took two approaches to narrow our focus on samples from 119 children at the times of most interest in this disease. First, we focused on all samples collected 120 between 0 and 2 months of age (when the majority of cases of FPIAP have presented). Second, 121 we made three key sample subsets: Last pre-symptomatic (where for each case of FPIAP we 122 chose their last pre-symptomatic sample and then the nearest age-matched control), First 123 symptomatic (where for each case of FPIAP we chose their first symptomatic sample and then 124 the nearest age-matched control), and First resolved (where for each case of FPIAP we chose 125 their first resolved sample and then the nearest age-matched control; Supplemental Figure 3).

126 In the 0-2 months sample subset, we again found a greater abundance of 127 Enterobacteriaceae_set1 $\left(p=1.54 \times 10^{-2}, \quad q=0.11, \quad c=0.140\right)$, and a lower abundance of 128 Enterobacteriaceae_set2 (unclassified at the genus level and most likely represented by 129 Klebsiella, Salmonella, or Enterobacter; Methods; $p=6.96 \times 10^{-2}, q=0.23, c=-0.095$ ), and an 130 unclassified family of Clostridiaceae $\left(p=5.83 \times 10^{-2}, q=0.22, c=-0.057\right)$ in FPIAP cases compared 131 to controls (Figure 3A). 
133 We next looked for taxonomic differences that might differentiate stages of FPIAP development 134 (pre-symptomatic, symptomatic, and resolved) from controls (Methods). Comparing infants with 135 FPIAP before they developed symptoms ("pre-symptomatic") to matched controls over the first 136 two months of life, we found a greater abundance of the genus Streptococcus $\left(p=5.26 \times 10^{-2}\right.$, $137 q=0.23, c=0.056)$ and Enterobacteriaceae_set1 $\left(p=1.89 \times 10^{-2}, q=0.16, c=0.15\right)$ and a lower 138 abundance of an unclassified family of Clostridiales $\left(p=3.41 \times 10^{-3}, q=5.21 \times 10^{-2}, c=-0.052\right)$ 139 (Figure 3B). Within the Last pre-symptomatic subset, we saw the same enrichment of 140 Enterobacteriaceae_set1 $(p=0.134, q=0.192, c=0.202)$ in infants with FPIAP (Figure 3A). Next 141 we looked at the symptomatic FPIAP cases, and found a greater abundance of 142 Enterobacteriaceae_set1 $\left(p=1.54 \times 10^{-2}, q=1.13 \times 10^{-1}, c=1.4 \times 10^{-1}\right)$, and a lower abundance of an 143 unknown family of Clostridiales $\left(p=4.8 \times 10^{-6}, q=1.27 \times 10^{-3}, \quad c=-6.33 \times 10^{-2}\right)$ in FPIAP cases 144 compared to controls over the first two months. The same enrichment of 145 Enterobacteriaceae_set1 and lower abundance of the same unclassified family of Clostridiales 146 was seen in the First symptomatic sample subset $\left(p=2.05 \times 10^{-3}, q=3.77 \times 10^{-2}, \quad c=0.217\right.$; $147 p=5.99 \times 10^{-6}, q=5.5 \times 10^{-4}, c=c=-0.087$ respectively) (Figure 3A). Finally, looking at infants with

148 FPIAP once their symptoms had resolved using samples from 9-12 months of age compared to 149 controls, we did not find taxonomic differences which met our coefficient and q-value threshold 150 (though differences were seen at a less stringent coefficient threshold) (Supplemental Figure 151 4). When we examined the more restrictive First resolved sample subset, we found an 152 enrichment of the genus Lactobacillus $\left(p=5.86 \times 10^{-3}, q=7.17 \times 10^{-2}, c=0.07\right)$ and a decreased 153 abundance of the genus Blautia $\left(p=1.22 \times 10^{-2}, q=0.105, c=-0.06\right)$ in infants with FPIAP which 154 had resolved compared to controls (Figure 3A).

155 Next, we were interested in the trajectories over time of each of these most significantly 156 differential taxa as the infants' disease progressed from pre-symptomatic, to symptomatic, to 
157 resolved states. Looking at all samples across the first year, the unknown family of the

158 Clostridiales class was significantly lower in children with FPIAP, before they became

159 symptomatic, while they had symptoms, and after they had resolved when compared to controls

160 (Figure 3C) with a relatively static trajectory. The relative abundance of the genus

161 Streptococcus was statistically higher than controls when infants with FPIAP were

162 presymptomatic, but then came down to levels comparable to controls once they developed

163 symptoms and then resolved (Figure 3C). Lactobacillus on the other hand, is lowest when

164 infants are presymptomatic (comparable to controls), rises sharply as they become

165 symptomatic, and then reaches a significantly higher abundance than controls when infants'

166 symptoms have resolved (Figure 3C). Infants with FPIAP were more likely to receive

167 Lactobacillus-containing probiotics, so we next examined whether this could explain the

168 differential abundance of Lactobacillus. Stratifying by probiotic exposure in the first year, we

169 found that while indeed infants receiving probiotics had a higher abundance of Lactobacillus, the

170 differential trajectory was seen in both groups, regardless of probiotic use (Supplemental

171 Figure 5). The Enterobacteriaceae_set1 also has an interesting trajectory: with a higher relative

172 abundance than controls even in the pre-symptomatic group, rising significantly higher than

173 controls as infants become symptomatic, and then decreasing but remaining higher than

174 controls even once symptoms have resolved (Figure 3C).

\section{Discussion:}

176 Food protein-induced allergic proctocolitis (FPIAP) is often the earliest manifestation of food

177 allergy in children, and yet little is known about its pathogenesis. Few studies have examined

178 the microbiome of infants with FPIAP, and to our knowledge none have dense longitudinal

179 sampling which includes samples prior to symptom onset. From the GMAP study we performed

180 16S rRNA gene sequencing on 954 longitudinal samples from 160 infants (81 with FPIAP and

18179 matched controls) from 1 week to 1 year of age. While overall the composition and richness

182 of the infants' microbiome was similar in infants with FPIAP compared to controls, we identified 
183 several taxonomic differences in infants with FPIAP across symptom states (from 184 pre-symptomatic to resolved).

185 We found an increased abundance of an unclassified genus of the Enterobacteriaceae family 186 which was most prominent when infants were symptomatic but was elevated even prior to 187 symptom onset. The Enterobacteriaceae family contains species known to bloom in other 188 inflammatory settings in the Gl tract ${ }^{26}$, and has previously been associated with cow's milk 189 allergy $^{15,16}$ and other food allergies ${ }^{27}$. We found a decreased abundance of an unclassified

190 family of Clostridiales. Some families within this order (Peptostreptococcaceae, 191 Lachnospiraceae, some Clostridium species) have been previously associated with decreased 192 relative abundance in IgE- and non IgE- food allergic children ${ }^{11,28,29}$ although many other 193 Clostridiales species are enriched. Previous reports suggest that specific species of Clostridia 194 drive the separation between allergic children and their non-allergic siblings and controls ${ }^{28}$. 195 Among these families are species which are important for gut permeability/integrity as well as 196 butyrate production, both of which have important potential mechanistic roles in FPIAP ${ }^{10,30}$. We 197 also found a greater abundance of members of the genus Lactobacillus as infants with FPIAP's 198 symptoms resolved compared to controls (irrespective of probiotic use). Other studies of infants 199 with a mix of IgE- and non-lgE-mediated cow's milk allergy have shown an increase in 200 Lactobacillus when infants were treated with hypoallergenic formula ${ }^{15}, \mathrm{FMT}^{31}$, or hypoallergenic 201 formula $+\mathrm{LGG}^{10}$. Finally, we found an unclassified genus of the Clostridiaceae family which was 202 significantly lower in the infants with FPIAP compared to controls overall, and similarly a lower 203 abundance of an unknown family of Clostridiales across symptom states in these allergic 204 infants. A few IgE-mediated food allergy studies have also shown decreased Clostridia 205 species $^{16,32}$ in food allergic subjects.

206 One limitation inherent to studying the microbiome of FPIAP is how early in infancy the disease 207 presents. The microbiome composition in the first few months of life is highly dynamic, making it 208 more difficult to detect differences between clinical groups. This likely accounts for some of our 209 findings which appeared in some sample subsets but not others, but also underscores the 210 significance of those findings that were replicated across time. The limited number of samples in 211 our presymptomatic sample subset, due to very young age, also likely limited our power to 212 identify differences in that subset. Another challenge to understanding FPIAP pathogenesis with 
213 respect to the microbiome is that diet often changes as infants are diagnosed (some with

214 maternal dietary elimination, some with changes to formula, some with changes across formula

215 types) which can have a significant impact on the microbiome and cannot be fully accounted for

216 in these models.

217 In summary, our unique longitudinal pediatric cohort enabled us to carefully investigate the role

218 of the microbiome in one of the earliest manifestations of allergy in children (FPIAP). Because of

219 the prospective healthy infant cohort design, we were able to identify key taxonomic differences

220 in children who developed FPIAP, even before they became symptomatic, as well as after their

221 disease resolved. We were also able to contribute to the growing body of literature describing

222 the early composition of the infant microbiome in non-allergic children and highlight several

223 potential areas of important further investigation. Our study provides a foundation for testable

224 hypotheses around the underlying role of potentially key taxa including Enterobacteriaceae and

225 Clostridiales in the pathophysiology of FPIAP. It remains important to further determine these

226 mechanisms as they may provide important opportunities for early targeted interventions to

227 prevent IgE and non-IgE-mediated food allergy more broadly, and perhaps to better support an

228 optimal pediatric microbiome promoting healthier non-allergic phenotypes.

\section{Methods:}

\section{Patient characteristics}

231 From the GMAP prospective observational healthy infant cohort, we selected infants diagnosed

232 with food protein-induced allergic proctocolitis (FPIAP) who had a minimum of 4 longitudinal

233 stool samples and selected matched controls for each who met the same sampling criteria. The

234 patient characteristics of this nested case-control subset were similar to those of the cohort at

235 large 6 .

\section{Sample collection}

237 Fresh stool samples were brought to each clinic visit, where stool was collected from diapers,

238 transferred to sterile tubes, stored immediately at $-20^{\circ} \mathrm{C}$, then transferred to $-80{ }^{\circ} \mathrm{C}$.

\section{DNA extraction and sequencing}

240 We used the Qiagen DNeasy PowerSoil htp 96 (Cat No./ID: 12955-4) to extract stool samples

241 that were stored at $-80 \mathrm{C}$ and "chipped" while frozen to obtain $100-150 \mathrm{mg}$ of sample for

242 extraction. Bead beating was performed on a Tissuelyzer at $20 \mathrm{~Hz}$ for $10 \mathrm{~min}$ as per

243 manufacturer's protocol.

244 16S rRNA gene libraries targeting the V4 region of the 16S rRNA gene were prepared by first

245 using qPCR to normalize template concentrations and determine optimal cycle number. Library

246 construction was performed in quadruplicate with the primers 515F

247 (5'-AATGATACGGCGACCACCGAGATCTACACTATGGTAATTGTGTGCCAGCMGCCGCGGTA 
248 A-3') and unique reverse barcode primers from the Golay primer set (Caporaso, 2011 and 2012). After amplification, sample replicates were pooled and cleaned via Agencourt AMPure XP-PCR purification system. Prior to final pooling, purified libraries were normalized via qPCR in two 25uL reactions, 2x iQ SYBR SUPERMix (Bio-Rad, REF: 1708880) with Read 1 (5'-TATGGTAATT GT GTGYCAGCMGCCGCGGTAA-3'), Read 2 (5'-AGTCAGTCAG CC ACTACNVGGGTWTCTAAT-3') primers. Pools were quantified by Qubit (Life Technologies, 254 Inc.) and sequenced on an Illumina MiSeq 300 using custom index 255 5'ATTAGAWACCCBDGTAGTCC GG CTGACTGACT-3' and custom Read 1 and Read 2 256 primers mentioned above.

257 Raw sequencing data can be found on NCBI BioProject PRJNA730851.

\section{$25816 \mathrm{~S}$ rRNA gene sequencing analysis}

259 Microbial communities were analyzed using QIIME2 version 2020.11.133. The DADA2 260 algorithm $^{34}$ was used for quality filtering and merging sequences with greater than $99 \%$ 261 similarity. Alignment was done by use of 'qiime feature-classifier fit-classifier-naive-bayes'. A 262 total of 1088 samples were collected. Samples that did not pass the sequencing process, had a 263 total frequency of less than 3000 reads, or those with an unclear diagnosis were dropped. 264 Additionally, features represented in only one sample or with a maximum relative abundance of $265<0.03$ were also dropped. A total of 954 samples remained for analysis. Relative abundance 266 data were normalised with QIIME2's tools. A reference Greengenes database version $13.5^{35}$ 267 was used to assign taxonomy features.

268 Some OTUs were not able to be classified on the genus, and were left as unclassified genera 269 within the Enterobacteriaceae family. For two of these sets that had significant results in our 270 data, we attempted to manually identify a better classification. We used blast-2.10.0+ to map the 271 OTU sequences against the NCBI nt database (downloaded on May 16 2017). We termed 272 these sets Enterobacteriaceae_set1, and Enterobacteriaceae_set2. Our results suggest that 273 Enterobacteriaceae_set1 is most likely representing sequences from the Escherichia genus, 274 while Enterobacteriaceae_set2 most likely represents sequences from the Klebsiella, 275 Salmonella and Enterobacter genera. In both sets there were many sequences that mapped to 276 "uncultured bacteria".

\section{Statistical analysis}

278 All analyses were performed in RStudio (Version 1.3.1093) run with $R$ (version 4.0.2). Data 279 editing done with dplyr. Graphs were created using R packages ggplot2 and ggpubr. Alpha diversity was calculated using the fossil package. Multivariate regression analysis was performed using the MaAsLin2 $\mathrm{R}$ package. Mixed-effects linear models using $a$ variance-stabilizing arcsin square root transform on relative abundances are then used to determine the significance of putative associations from among this reduced set. Nominal $P$ values across all associations were then adjusted using the Benjamini-Hochberg FDR method. We reported microbial features with corrected $q<0.25$ were reported. $p$ values that did not originate from the MaAsLin analysis were generated using the rstatix $\mathrm{R}$ package.

All boxplots were generated using ggplot2: The lower and upper hinges correspond to the first and third quartiles (the 25th and 75th percentiles), and the upper and lower whiskers roughly represent the $1.5^{\star} I Q R$ from the hinge (default geom_boxplot function). 
The composition and richness analysis were calculated using a subset of 808 samples, such that each infant has a single sample at each time point. Richness was calculated using the chao1 function in the fossil $R$ package.

\section{Multivariate models definition}

295 To find a linear correlation between FPIAP and microbial features we used three different 296 models: (1) comparing samples from FPIAP cases to controls; (2) comparing samples based on their allergic symptoms (pre-symptomatic, symptomatic, and resolved) to controls; and (3) as in (2) but comparing the samples to the symptomatic group as a reference. Each model contains the following fixed effects variables: (a) case/control or symptoms (b) mode of delivery (c) age at visit (d) diet and (e) probiotics use in the first year of life. As we have multiple samples from each child, all models also include the child ID as a random variable.

In general, we ran the models on six different subsets of samples, either subsetting by age or by disease status:

Age subgroups -
a. All Samples - This group includes all samples ( $n=954)$.

b. $0-2$ months - All samples with age visit $<3$ months $(n=473)$.

c. 9-12 months - All samples with age visit in the range [8.5-12.5] months $(n=179)$.

Disease status subgroups (these subsets always contain a single sample per subject) -

d. Last pre-symptomatic - For each FPIAP case we chose the last sample collected prior to symptom onset, and identified a matched control sample $(n=114)$.

e. First symptomatic - For each FPIAP case we chose the first sample when the child was symptomatic, and identified a matched control sample $(n=144)$.

f. First resolved - For each FPIAP case we chose the first sample when the child was asymptomatic, and identified a matched control sample $(n=148)$.

Matched control samples were chosen by finding an optimal match between the FPIAP sample times and all the control samples, making sure to include at most a single sample from each control subject in each subset. We found the optimal match by defining it as a linear sum assignment problem (LSAP) using the clue R package to solve it with the hungarian method.

\section{Interactive visualization tool}

All associations (significant and not significant) can be viewed on our interactive visualization tool: https://www.cs.huji.ac.il/ morani/GMAP data.html, enabling the comparison of multiple models, and features, together with viewing the raw data and the statistical analysis results.

\section{References: (not more than 70)}

1. Ege, M. J. et al. Exposure to environmental microorganisms and childhood asthma. N. Engl. J. Med. 364, 701-709 (2011).

2. Prince, B. T., Mandel, M. J., Nadeau, K. \& Singh, A. M. Gut Microbiome and the Development of Food Allergy and Allergic Disease. Pediatr. Clin. North Am. 62, 1479-1492 
(2015).

3. Hooper, L. V., Littman, D. R. \& Macpherson, A. J. Interactions between the microbiota and the immune system. Science 336, 1268-1273 (2012).

4. Okada, H., Kuhn, C., Feillet, H. \& Bach, J.-F. The 'hygiene hypothesis' for autoimmune and allergic diseases: an update. Clin. Exp. Immunol. 160, 1-9 (2010).

5. Bach, J.-F. The effect of infections on susceptibility to autoimmune and allergic diseases. $N$. Engl. J. Med. 347, 911-920 (2002).

6. Martin, V. M. et al. Prospective Assessment of Pediatrician-Diagnosed Food Protein-Induced Allergic Proctocolitis by Gross or Occult Blood. J. Allergy Clin. Immunol. Pract. 8, 1692-1699.e1 (2020).

7. Martin, V. M. et al. Increased IgE-Mediated Food Allergy With Food Protein-Induced Allergic Proctocolitis. Pediatrics 146, (2020).

8. Mennini, M. et al. Food protein-induced allergic proctocolitis in infants: Literature review and proposal of a management protocol. World Allergy Organ. J. 13, 100471 (2020).

9. Radano, M. C. et al. Cesarean section and antibiotic use found to be associated with eosinophilic esophagitis. J. Allergy Clin. Immunol. Pract. 2, 475-477.e1 (2014).

10. Berni Canani, R. et al. Gut microbiota composition and butyrate production in children affected by non-lgE-mediated cow's milk allergy. Sci. Rep. 8, 12500 (2018).

11. Ling, Z. et al. Altered fecal microbiota composition associated with food allergy in infants. Appl. Environ. Microbiol. 80, 2546-2554 (2014).

12. Nevoral, J. et al. Intestinal microbiota in exclusively breast-fed infants with blood-streaked stools. Folia Microbiol. 54, 167-171 (2009).

13. Kumagai, $H$. et al. Intestinal microbiota and secretory immunoglobulin $A$ in feces of exclusively breast-fed infants with blood-streaked stools. Microbiol. Immunol. 56, 657-663 (2012).

14. Berni Canani, R. et al. Lactobacillus rhamnosus GG-supplemented formula expands butyrate-producing bacterial strains in food allergic infants. ISME J. 10, 742-750 (2016).

15. Dong, P., Feng, J.-J., Yan, D.-Y., Lyu, Y.-J. \& Xu, X. Early-life gut microbiome and cow's milk allergy- a prospective case - control 6-month follow-up study. Saudi J. Biol. Sci. 25, 875-880 (2018).

16. Bunyavanich, S. et al. Early-life gut microbiome composition and milk allergy resolution. J. Allergy Clin. Immunol. 138, 1122-1130 (2016).

17. Guadamuro, L. et al. Fecal Changes Following Introduction of Milk in Infants With Outgrowing Non-lgE Cow's Milk Protein Allergy Are Influenced by Previous Consumption of the Probiotic LGG. Front. Immunol. 10, 1819 (2019).

18. Díaz, M. et al. Microbiota and Derived Parameters in Fecal Samples of Infants with Non-lgE Cow's Milk Protein Allergy under a Restricted Diet. Nutrients 10, (2018).

19. Bäckhed, F. et al. Dynamics and Stabilization of the Human Gut Microbiome during the First Year of Life. Cell Host Microbe 17, 690-703 (2015).

20. Yassour, M. et al. Natural history of the infant gut microbiome and impact of antibiotic treatment on bacterial strain diversity and stability. Sci. Transl. Med. 8, 343ra81 (2016).

21. Cher, A. \& Yassour, M. The compositional development of the microbiome in early life. in The Human Microbiome in Early Life 177-195 (Elsevier, 2021).

22. Penders, J. et al. Factors influencing the composition of the intestinal microbiota in early infancy. Pediatrics 118, 511-521 (2006).

23. Yatsunenko, T. et al. Human gut microbiome viewed across age and geography. Nature 486, 222-227 (2012).

24. Mitchell, C. M. et al. Delivery Mode Affects Stability of Early Infant Gut Microbiota. Cell Rep Med 1, 100156 (2020).

25. Rutayisire, E., Huang, K., Liu, Y. \& Tao, F. The mode of delivery affects the diversity and colonization pattern of the gut microbiota during the first year of infants' life: a systematic 
review. BMC Gastroenterol. 16, 86 (2016).

26. Zeng, M. Y., Inohara, N. \& Nuñez, G. Mechanisms of inflammation-driven bacterial dysbiosis in the gut. Mucosal Immunol. 10, 18-26 (2017).

27. Azad, M. B. et al. Infant gut microbiota and food sensitization: associations in the first year of life. Clin. Exp. Allergy 45, 632-643 (2015).

28. Kourosh, A. et al. Fecal microbiome signatures are different in food-allergic children compared to siblings and healthy children. Pediatr. Allergy Immunol. 29, 545-554 (2018).

29. Feehley, T. et al. Healthy infants harbor intestinal bacteria that protect against food allergy. Nat. Med. 25, 448-453 (2019).

30. Wlodarska, M. et al. Indoleacrylic Acid Produced by Commensal Peptostreptococcus Species Suppresses Inflammation. Cell Host Microbe 22, 25-37.e6 (2017).

31. Liu, S.-X. et al. Fecal microbiota transplantation induces remission of infantile allergic colitis through gut microbiota re-establishment. World J. Gastroenterol. 23, 8570-8581 (2017).

32. Hua, X., Goedert, J. J., Pu, A., Yu, G. \& Shi, J. Allergy associations with the adult fecal microbiota: Analysis of the American Gut Project. EBioMedicine 3, 172-179 (2016).

33. Bolyen, E. et al. Reproducible, interactive, scalable and extensible microbiome data science using QIIME 2. Nat. Biotechnol. 37, 852-857 (2019).

34. Callahan, B. J. et al. DADA2: High-resolution sample inference from Illumina amplicon data. Nat. Methods 13, 581-583 (2016).

35. McDonald, D. et al. An improved Greengenes taxonomy with explicit ranks for ecological and evolutionary analyses of bacteria and archaea. ISME J. 6, 610-618 (2012). 
Acknowledgements: We thank Tiffany Poon and Luke Besse for project management, the staff

414 final manuscript.

\section{Figure Legends:}

\section{Figure 1. GMAP Study Cohort design, key clinical characteristics, and longitudinal}

417 sampling. (A) Key clinical and environmental features of the cohort analyzed including antibiotic 418 exposure, mode of delivery, and infant diet over time and the timeline for sample collection. (B) 419 Number of samples analyzed across the first year, binned by the age of the infant at the time of 420 sample collection and colored by whether that infant was a healthy control or a child with FPIAP.

421 Samples from infants with FPIAP are colored by their symptom state at the time of sample 422 collection. (C): Sample map showing the samples analyzed plotted by the age of the infant at 423 time of collection and colored by their disease and symptom state. 
424 Figure 2. Overall longitudinal microbiome composition. (A) Composition plot of the full first 425 year of life showing the mean relative abundance of the top 15 taxa and their longitudinal 426 taxonomic assemblage over the first year (Methods). (B) Longitudinal microbial richness (using 427 the chao1 index) over the first year. The center line denotes the median, the boxes cover the 428 25th to 75th percentiles (see Methods). (C) Relative abundance (arcsine transformed, AST) of 429 key taxonomic differences mediated by important environmental factors: Bacteroides by delivery 430 mode, Bifidobacterium by infant diet, and Lactobacillus by probiotic use. Significant differences 431 and coefficients from MaAsLin 2 model across all samples (see Methods).

432 Figure 3. Summary of key differential taxa between infants with FPIAP and healthy 433 controls. (A) Significantly different taxa $(q<0.25)$, with absolute coefficient $>=0.05$, when 434 comparing infants with FPIAP to healthy controls when looking at the sample subsets: $0-2$ 435 months, Last pre-symptomatic, First symptomatic, and First resolved. Bars to the right are 436 enriched in infants with FPIAP, while bars to the left are enriched in the controls. (B) 437 Significantly different taxa $(q<0.25)$ when comparing infants with FPIAP to matched controls 438 before their symptom onset (top section), and then during the symptomatic period (lower 439 section) over the first 2 months of age. Association directionality is as in (A). (c) Relative 440 abundance trajectories of the key differential taxa between cases and controls identified in 441 panels $(A)$ and $(B)$ across symptom states (from pre-symptomatic to symptomatic to resolved).

442 Supplemental Figure 1. Top 15 abundant taxa in infants with FPIAP compared to controls.

443 Composition plots showing the mean relative abundance of the top 15 abundant taxa and their 444 longitudinal taxonomic assemblage over the first year in infants with FPIAP (left) compared to 445 controls (right).

446 Supplemental Figure 2. Alpha diversity in infants with FPIAP compared to controls 447 examined longitudinally over the first year by symptom state. Alpha diversity measured by 
chao1 richness index in control infants compared to infants with FPIAP before symptom onset,

449 during the symptomatic period, and after symptom resolution over the first year.

450 Supplemental Figure 3. Sample map. Sample map showing longitudinal samples used for 451 each subset analyzed, axes and colors as in Figure 1.

\section{Supplemental Figure 4. Summary of additional key differential taxa between infants with}

453 FPIAP and healthy controls. Same analysis as Figure 2, with less stringent coefficient 454 threshold (>0.025). (A) Significantly different taxa when comparing infants with FPIAP to healthy 455 controls when looking at the sample subsets: 0-2 months, Last pre-symptomatic, First 456 symptomatic, and First resolved. Bars to the right are enriched in infants with FPIAP, while bars 457 to the left are enriched in the controls. (B) Significantly different taxa when comparing infants 458 with FPIAP to matched controls before their symptom onset (top section), and then during the 459 symptomatic period (lower section) over the first 2 months of age. Again, bars to the right are 460 enriched in infants with FPIAP, while bars to the left are enriched in the controls.

461 Supplemental Figure 5. Differential trajectory of Lactobacillus across symptom states in 462 infants with FPIAP compared to controls, stratified by probiotic use. Box plots of the 463 relative abundance (AST) trajectories of Lactobacillus across symptom states (from 464 pre-symptomatic to symptomatic to resolved) compared to controls, stratified by (largely 465 LGG-containing) probiotic use (panel A vs. panel B) across all samples.

466 Supplemental Table 1. Summary table of all significant MaAsLin results. All MaAsLin 467 results from 16S rRNA gene sequencing with q-values for significant taxa which met our 468 predetermined threshold for significance $(q<0.25)$. Mixed effects linear models using arcsine 469 transform on relative abundances were used to determine significance (see Methods). p-values 470 were adjusted for multiple comparisons using the Benjamini-Hochberg false discovery rate 471 (FDR) method with FDR set at $<0.25$. 
Figures
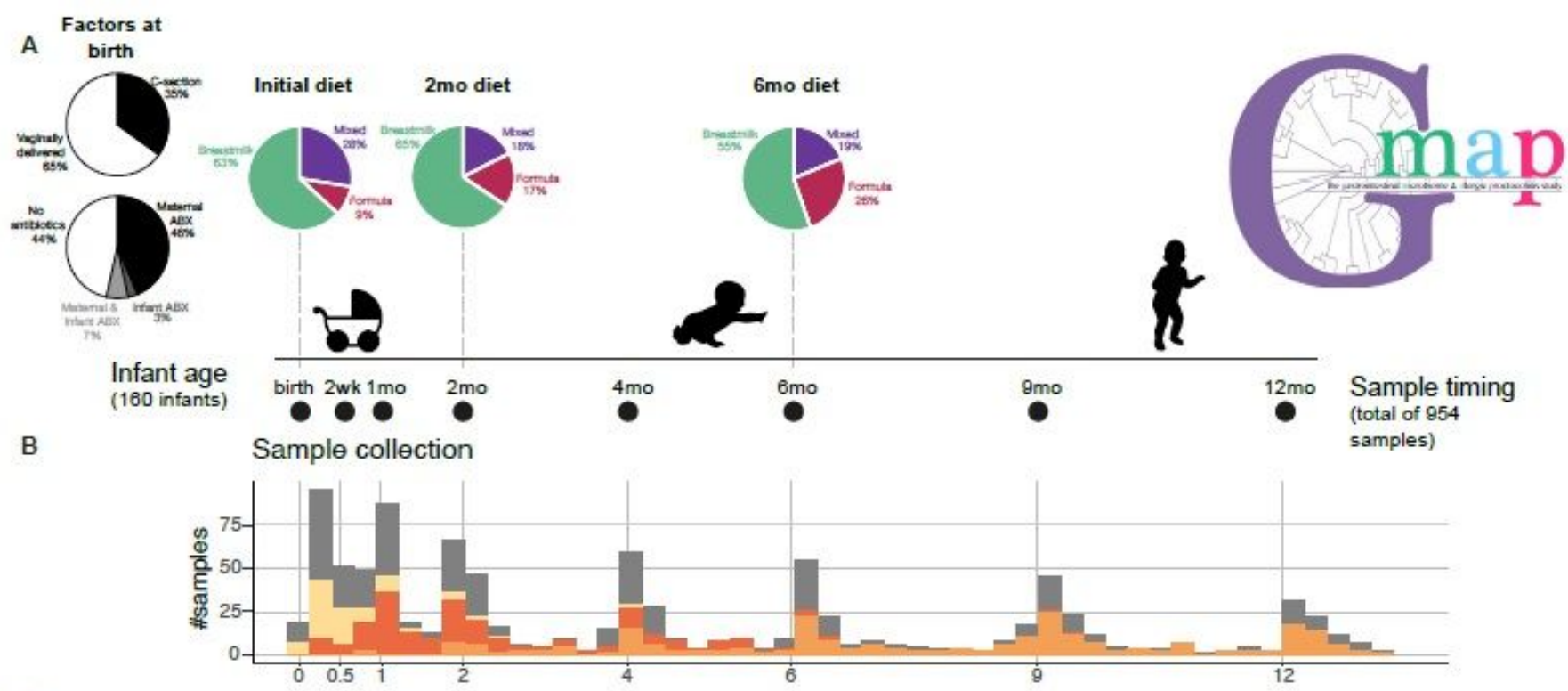

C

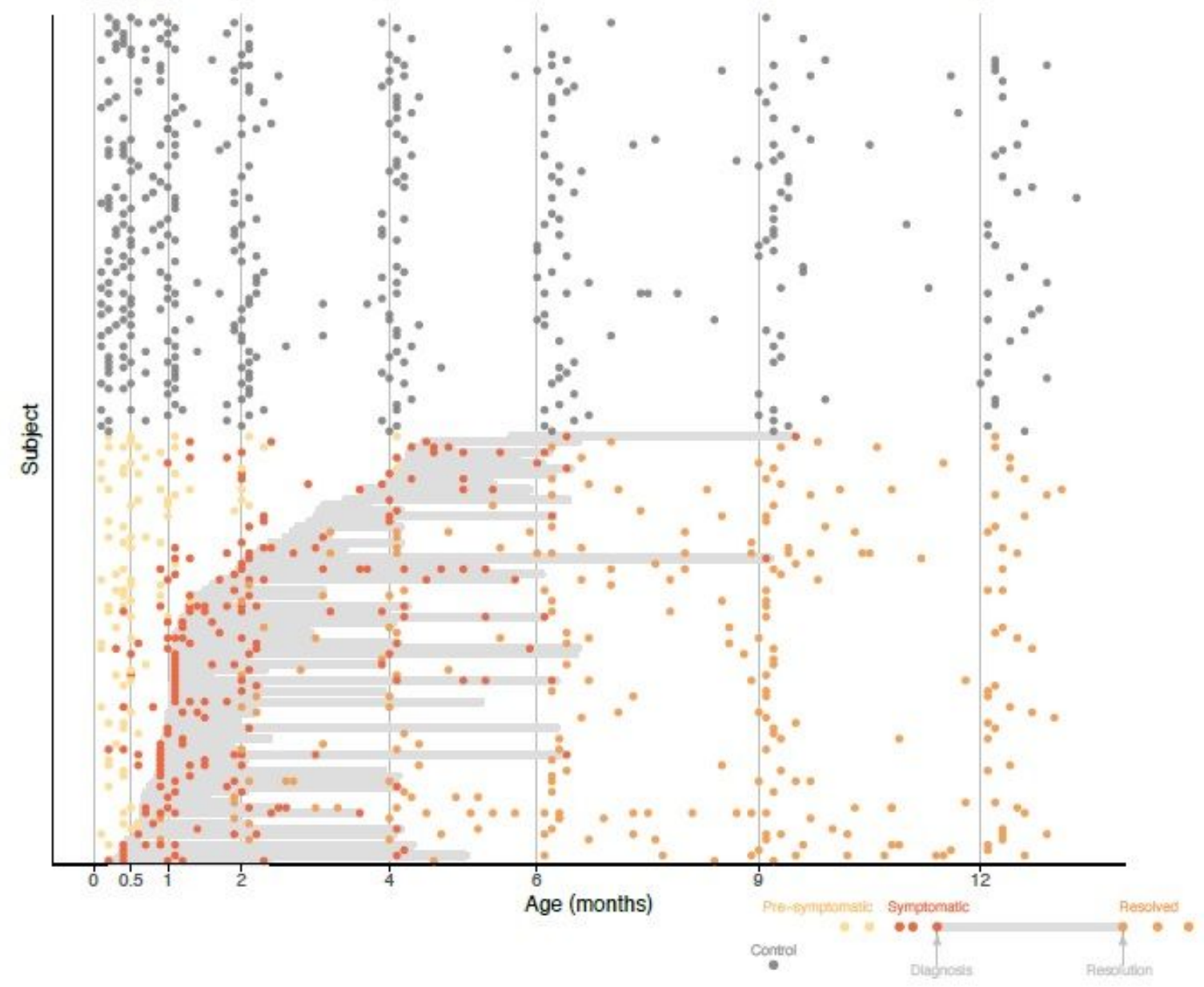

Figure 1

GMAP Study Cohort design, key clinical characteristics, and longitudinal sampling. (A) Key clinical and environmental features of the cohort analyzed including antibiotic exposure, mode of delivery, and infant diet over time and the timeline for sample collection. (B) Number of samples analyzed across the first 
year, binned by the age of the infant at the time of sample collection and colored by whether that infant was a healthy control or a child with FPIAP. Samples from infants with FPIAP are colored by their symptom state at the time of sample collection. (C): Sample map showing the samples analyzed plotted by the age of the infant at 423 time of collection and colored by their disease and symptom state.

A
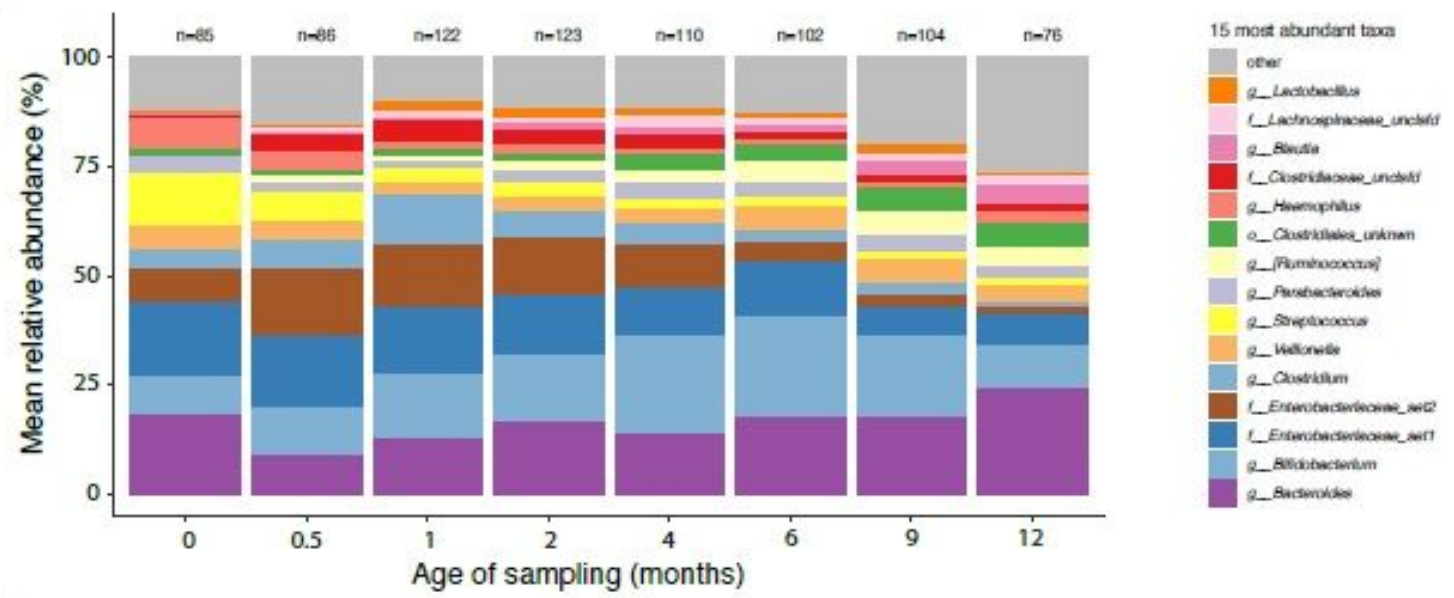

B

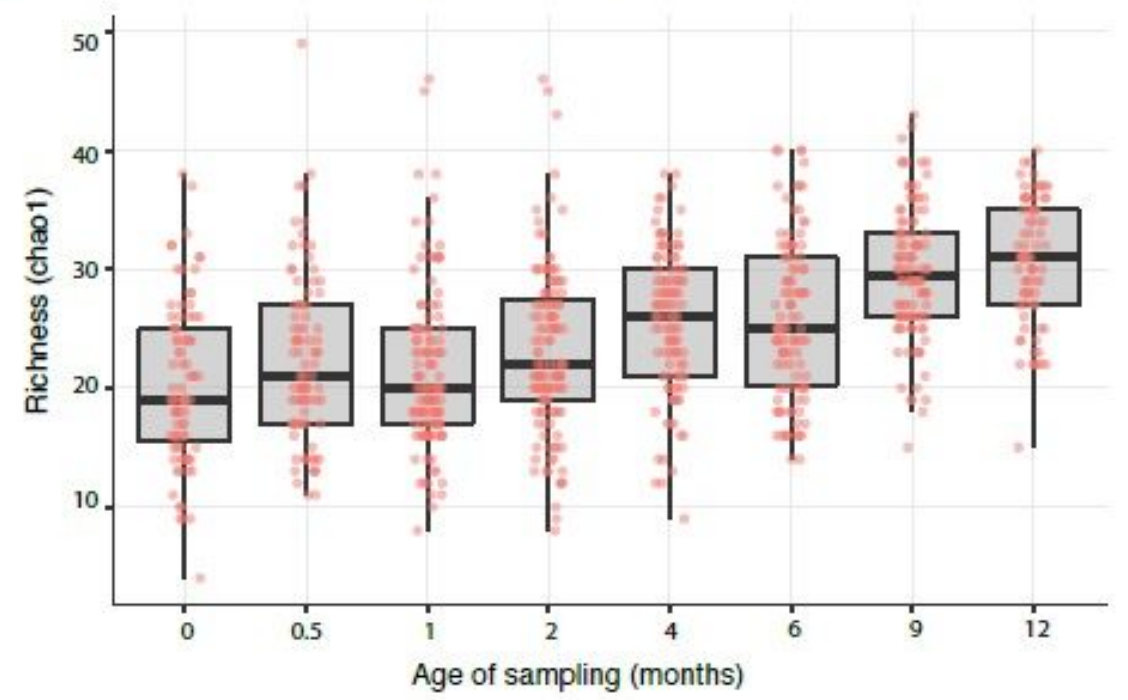

C
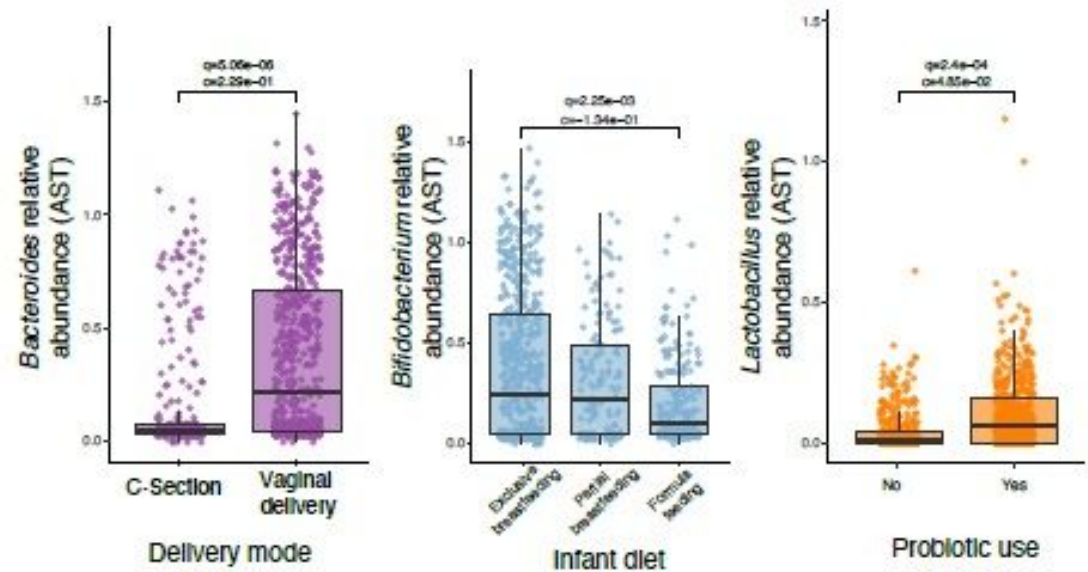

Figure 2 
Overall longitudinal microbiome composition. (A) Composition plot of the full first year of life showing $t$ he mean relative abundance of the top 15 taxa and their longitudinal taxonomic assemblage over the first year (Methods) . (B) Longitudinal microbial richness (using the chao1 index) over the first year. The center line denotes the median, the boxes cover the 25th to 75th percentiles (see Methods) . (C) Relative abundance (arcsine transformed, AST) of key taxonomic differences mediated by important environmental factors: Bacteroides by delivery mode, Bifidobacterium by infant diet, and Lactobacillus by probiotic use. Significant differences and coefficients $\mathrm{f}$ rom MaAsLin 2 model across all samples (see Methods).

A
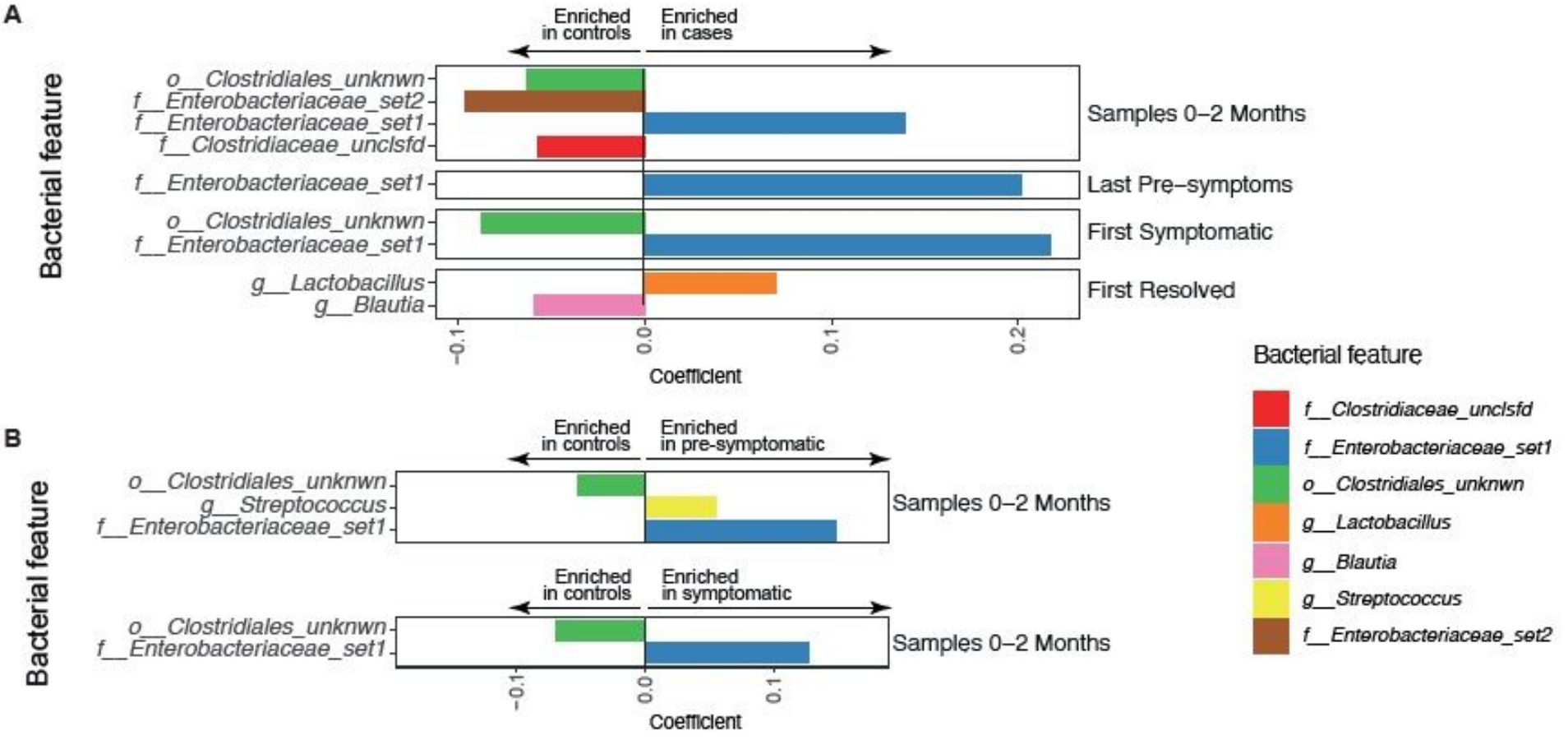

C

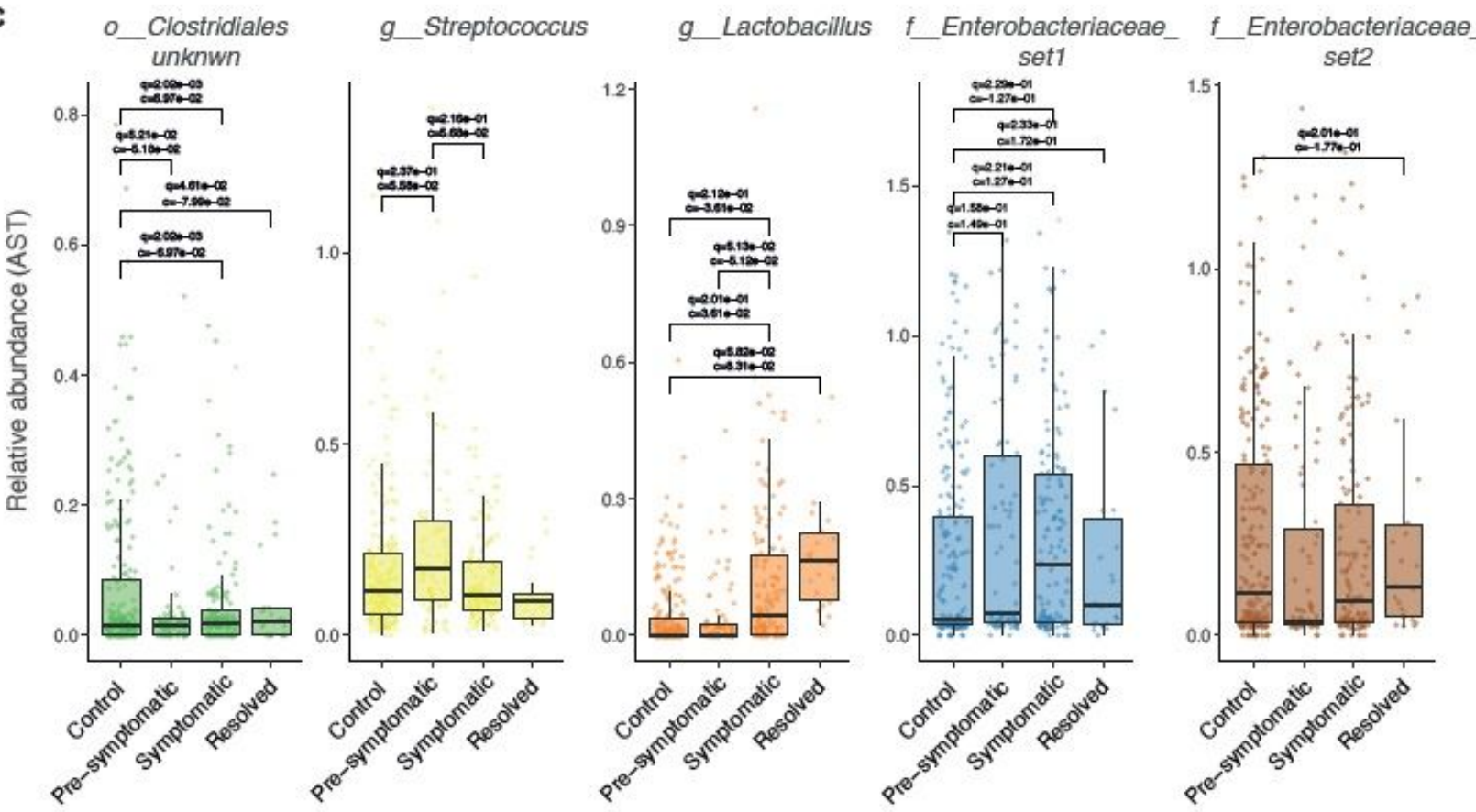

Figure 3 
Summary of key differential taxa between infants with FPIAP and healthy controls. (A) Significantly different taxa $(q<0.25)$, with absolute coefficient $>=0.05$, when comparing infants with FPIAP $t o$ healthy controls when looking at the sample subsets: 0-2 months, Last pre-symptomatic, First symptomatic, and First resolved. Bars to the right are enriched in infants with FPIAP, while bars to the left are enriched in the controls. (B) Significantly different taxa $(\mathrm{q}<0.25)$ when comparing infants with FPIAP to matched controls before their symptom onset (top section), and then during the symptomatic period (lower section) over the first 2 months of age. Association directionality is as in (A). (C) Relative abundance trajectories of the key differential taxa between cases and controls identified in panels (A) and (B) across symptom states (from pre-symptomatic to symptomatic to resolved).

\section{Supplementary Files}

This is a list of supplementary files associated with this preprint. Click to download.

- PublishResultsFromSuppTable1.xlsx

- SuppFiguresclean.pdf 